\title{
Views of Male Community Elders With Regards to Medical Male Circumcision at Pfanani Clinic in Limpopo Province, South Africa
}

\author{
T. M. Mothiba ${ }^{1} \&$ M. A. Bopape ${ }^{2}$ \\ ${ }^{1}$ Faculty of Health Sciences, Dean's office, University of Limpopo, South Africa \\ ${ }^{2}$ Department of Nursing Science, University of Limpopo, South Africa \\ Correspondence: Tebogo Mothiba, Faculty of Health Sciences, University of Limpopo, Private Bag X1106 \\ Sovenga 0727, South Africa. Tel: 015-267-3080; Fax: 015-268-2387. E-mail: tebogo.mothiba@ul.ac.za
}

Received: January 2, 2019 Accepted: March 6, 2019 Online Published: May 20, 2019

doi:10.5539/gjhs.v11n6p193

URL: https://doi.org/10.5539/gjhs.v11n6p193

\begin{abstract}
The purpose of this study was to explore the views of male community elders to Medical Male Circumcision at Pfanani clinic in Limpopo Province. A qualitative, descriptive and explorative research design was used. Purposive sampling was used whereby the researchers interviewed a total of 18 male community elders of ages ranging from 40 to 75 years who came for consultation at the Pfanani clinic. Data was collected using semi-structured one to one interviews. Data were analyzed using the Tesch's open-coding method one theme and its sub-themes emerged. The study found that Medical Male Circumcision is sometimes not a safe procedure, there are several unexpected outcomes which are experienced, Traditional Male Circumcision predispose males to infections, the environment where the Traditional Male Circumcision is done predisposes males to bad environmental conditions, the period when the Traditional Male Circumcision is performed is viewed as a moral teaching session and lessons learned prepares boys for manhood. The study recommended that the Department of Health should conduct workshops and training for traditional circumcisers and train them on how to maintain sterility during circumcision to avoid the initiates not having infection.
\end{abstract}

Keywords: views, community elders, medical male circumcision

\section{Introduction and Background}

Medical Male Circumcision (MMC) is described by Morris, Bailey, Klausner et al. (2015) as a simple, low-risk procedure with very little or no adverse long-term effect on sexual function, sensitivity, sensation during arousal or overall satisfaction. Medical male circumcision is an effective circumcision practice found in many parts of the world that is provided privately, voluntarily and with counseling offered before the procedure can be done (Wambara, Mwanga, Mosha, Mshana, Mosha, \& Changalucha, 2011). Male medical circumcision has been pronounced especially amongst Jews, Arabs, Australians Aborigines, Indonesians, Malaysians and Americans (WHO UNAIDS, 2010).

The practice of male circumcision is rationalized differently by various cultures. Some cultures consider it a cosmetic procedure. Amongst the Jewish community, circumcision of male Jewish babies on the eighth day after birth represents part of Abraham's covenant with God (WHO and UNAIDS, 2010). Male circumcision in other societies is performed at the beginning of adolescence as a rite of passage to adulthood, the circumcision festival is used also to educate young men about various health issues, including sexual and reproductive health. These cultural traditions can be harmonized with modern clinical practice, to ensure the safety of circumcision and to use the opportunity to educate young men about a number of sexual and reproduction health issues (WHO \& UNAIDS, 2008). In Uganda, like in most sub-Saharan countries, traditional male circumcision is practiced and it marks the entry to manhood (Sarvestani, Bufumbo, Gerger, \& Sienko, 2012). In South Africa, the Xhosa tribe consider male circumcision as a sacred religious custom which symbolically transforms boys into respected men in the community. The circumcised men have power and authority in the community over women and uncircumcised men (Mavundla, Netswera, Toth, Botttomon, \& Tenge, 2010).

Male circumcision in other cultures is performed at various growths and development stages during lifespan which affects the overall health and well-being of males (Mavundla et al., 2010). There is a difference among different African ethnic groups in traditional male circumcision rates, whereby the age of traditional circumcision for 
Vhavenda ranges from 6 years to 50 years (Peltzer, 2009). The study conducted by Tarimo, Francis, Kakoko, Munseri, Bakari and Sandstrom (2012), indicated that respondents are worried about circumcision of older people and they think the integration of male circumcision services in the routine childhood vaccination programme may increase coverage during the infancy period.

The elders at Nyaza village in the Western province of South Africa, home of the Babukusu, view traditional male circumcision as the best as compared to medical male circumcision. The procedure is performed in public by a traditional surgeon who has no formal training. The procedure is often painful and it's an essential part of the ceremony that the boy experience pain in order to become a man. Elders also view circumcision as the initiation of a boy into the rights and responsibilities of manhood. No man should marry until he has been circumcised and it is considered an embarrassment, if not taboo, to wed before being circumcised. It is a symbol of courage and represents a boy's respect for his family and community, and bringing his relative's honor (Bailey, Moses, Parker et al., 2007). Traditional male circumcision in some countries takes place in circumcision camps or ceremonies were traditional circumcisers performed the procedure to groups of boys without anesthesia. The event is usually festive and educational for participants and the community. The goals are to acknowledge the boys' physical and emotional maturity and readiness to face the challenges of adulthood, as well as support them during the painful circumcision procedure (WHO \& UNAIDS, 2008).

Medical male circumcision is perceived as a replacement of traditional male circumcision in the context of HIV prevention (Gwandure, 2011). Medical Male Circumcision (MMC) is an integral part of the HIV prevention programme in South Africa and it has led to an estimated 60\% decrease in female-to-male HIV transmission (WHO \& UNAIDS, 2007). The World Health Organisation (WHO) and the Joint United Nations Programme on AIDS recommends male circumcision as an additional intervention against HIV infection transmission. Most men who are not circumcised suffer a medical condition caused by their foreskins (Morries \& Cox, 2012). Voluntary male medical circumcision is recommended by WHO, to be the key component of prevention strategies in settings where there is high HIV prevalence. The greatest reduction in HIV incidence can be achieved if $80 \%$ of HIV negative males aged between 15 and 49 can be circumcised with the potential to prevent new HIV infections in South Africa (Kripke et al., 2016).

In Limpopo province of South Africa, traditional male circumcision is mainly practiced among African Black racial groups. Although there is a difference among different African ethnic groups with regard to male circumcision, where most community elders value traditional male circumcision than Medical Male Circumcision and seem to be discouraging men to go for MMC. Traditional Male Circumcision is referred to as a symbol of courage and represents a boy's respect for his family and community, and bringing his relative's honor (Bailey et al., 2007). Despite the community elder's value of TMC, MMC uptake is increasing at the Pfanani clinic with statistics increase from 10 bookings to 20 bookings for MMC from October to December 2014. Based on this background, the study seeks to determine the views of community elders to medical male circumcision.

\subsection{Study Site}

The area of the study is the Pfanani clinic which is situated at Ngovhela-Phindula Village under Sibasa Local area of Thohohandou at Vhembe district Limpopo Province. Pfanani clinic is 10 kilometers from N1 Road, then R524 of Thohoyandou which connects to R523.

\section{Research Design}

The qualitative research approach was employed to conduct the study. Qualitative research attempts to understand the phenomenon in it's entire, rather than focusing on specific concepts (Brink, Van der Walt, \& Rensburg, 2012). The qualitative research allows the researchers to obtain an in-depth understanding concerning the views of the community elders regarding Medical Male Circumcision.

\subsection{Exploratory Design}

The explorative design was used to explore the phenomenon studied (Brink et al., 2012). The researcher explored. The researcher asked one central question and probing questions were asked following a response to a central question in order to elicit more information related to the views of community elders' in relation to Medical Male Circumcision. The central question which was asked to all the participants, in the same way, was "Can you describe your views related to Medical Male Circumcision".

\subsection{Descriptive Design}

A descriptive design was aimed at obtaining complete and accurate information about a phenomenon through observation, description, and classification. The researcher gave the participants the opportunity to describe their 
views related to Medical Male Circumcision through semi-structured interviews.

\subsection{Population and Sampling}

The population was all community elders who came for consultation at the Pfanani clinic of Limpopo province. A non-probability purposive sampling technique was used in this study, where male elders who had information regarding male circumcision were recruited from the Pfanani clinic and voluntarily participated in the study (Babbie \& Mouton, 2012). The number of participants recruited was based on data saturation, and data collection was stopped when no new information emerged from the interviews. A total of 18 community elders eligible for participation included were of ages ranging from 40 to 75 years who came for consultation at Pfanani clinic. The community elders were residences of Sibasa local area of the Vhembe district of Limpopo.

\subsection{Data Collection Method}

In this study, semi-structured in-depth interviews with an interview guide were conducted to enable participants to express their personal views on MMC for a period of four weeks at Pfanani clinic. The interview sessions were conducted in a private room away from distractions. One central question was asked, "Could you kindly describe your views related to medical male circumcision?" This question was followed by clarity seeking questions to probe after the first response of each participant. Field notes were captured for non-verbal communication and voice recorder was used to capture all the interview sessions.

\subsection{Data Analysis}

The data analysis method that was used in this study was Tesch's open coding method which comprises of eight integrated steps as stated in (Creswell, 2009). Recordings were transcribed verbatim by the researchers and then translated to English. The researchers familiarised themselves with data by reading through transcriptions repeatedly to identify key ideas to get a sense of the interviews conducted as a whole. A list of all topics and similar topics were grouped together and were arranged in major, unique topics and leftovers and then compared with the original data. The researchers abbreviated the topics as codes and codes were written next to the appropriate segment of the text. A summary of the themes and sub-themes identified is presented in Table 1.

\subsection{Measures to Ensure Trustworthiness}

In this study, three criteria to ensure trustworthiness, as outlined by de Vos, Strydorm, Fouché, and Delport (2011), were used. Credibility was ensured through prolonged engagement with the participants and the interview sessions lasted for approximately 50 minutes to an hour and for a period of two weeks. Credibility and confirmability were ensured by the utilization of the voice recorder to record all interview sessions. Transferability was ensured through a complete description of the research design and methodology used and also through the purposive selection of participants. Voice recorder and field notes responses were kept as part of the audit trail and added to the triangulation of data to ensure credibility and confirmability.

\subsection{Ethical Considerations}

Ethical clearance was obtained from the University of Limpopo Research Ethics Committee (TREC) and the permission to collect data was sort with the Department of Health Limpopo province and the manager of the Pfanani clinic.

The researcher explained the purpose and objective of the study to every participant before the commencement of the interviews. The participants were made aware of what is expected from them during interview sessions regarding their participation which was only to answer questions asked, and it was also indicated that they are not forced to do so if they do not feel comfortable. The participants were informed that their participation is voluntary and they can withdraw from participating during the data collection session without any penalty at any time. The participants were informed that field notes will be written and a voice recorder will be used to capture all the interview sessions.

The principle of autonomy was ensured by allocating each one code instead of the participant's name in the study with the aim of not revealing the participants' identity.

\section{Results}

The results of this study are based on data collected during unstructured interview sessions conducted with community elders at Mfanani clinic and data were analysed using Tesch's open coding methods as described by Creswell (2009). A theme and four sub-themes related to views of elders in relation to Medical Male Circumcision have emerged from data analysis. 
Table 1. Theme and sub-themes

\begin{tabular}{|c|c|}
\hline Theme & Sub-Themes \\
\hline Views of elders related to Medical Male Circumcision & $\begin{array}{l}\text { - } \text { MMC viewed as the commendable method } \\
\text { - } \quad \text { Safety is guaranteed } \\
\text { - } \quad \text { MMC viewed as undermining the black culture } \\
\text { - } \quad \text { Less pains experienced }\end{array}$ \\
\hline
\end{tabular}

\section{Theme: Views of elders related to medical male circumcision.}

The findings have one theme that is the views of male elders related to traditional male circumcision and four sub-themes that have emerged under this theme. The sub-themes are discussed and direct participants excerpts are presented in italic to support the findings. Literature is also presented to support the findings with studies conducted.

\section{Sub-theme: Male medical circumcision as a commendable method}

The findings revealed that medical male circumcision is a commendable method to men who believe in modern ways of doing things, this was confirmed by Elder 2 who said: ... "To sum it up, I will prefer my kids to be circumcised in a clinic or hospital". Elder 1 with the same opinion said: ... "And as such to me the modern medical male circumcision is the best as the safety of initiates' lives are guaranteed". Elder 4 also added by saying:... "By the look of things medical male circumcision is the best, safest and the cheapest, I even sent my two boys to the clinic to be circumcised, believe me, it was a painless easy experienced". In support of the findings, Sgaier, Reed, Thomas, and Njeuhmeli (2014) indicated that Medical Male Circumcision which is voluntary in nature reduces the risk of sexually transmitted diseases, therefore, it is recommended.

\section{Sub-theme: Safety is guaranteed}

The findings of this present study revealed that participants viewed Medical male circumcision as a safe procedure and this was evidenced by elder 6 who said..."Medical male circumcision gives a lot of benefits to young men attended by highly trained medical practitioners and they went through clean safe operation". Elder 8 said that..."Medical male circumcision is good to numerous reasons were the lives of young boys are guaranteed because of its safety". Elder 1 also shared the same opinion by saying: ... "And as such to me the modern medical male circumcision is the best as the people who do this procedure at the hospital come home alive. We know that there is no way a person will not come home but with the one that people go to the mountains, it is not safe at all. Our children die in the mountains in the mountains." Elder 5 verbalized that "My children refuse to send their children to the mountains because they are afraid something can happen to them in the mountains. But with rate things are happening I cannot differ with them". Medical male circumcision is conducted by a senior health care provider in a clean and hygienic medical facility (WHO, 2009).

\section{Sub-themes: Medical male circumcision viewed as undermining the black culture}

The findings of this present study revealed that male medical circumcision undermine the black culture this was evidenced by elder 14 who said..." in my opinion as an elder who values my custom and tradition this thing medical circumcision is all for white people who now take advantage of our knowledge and makes us believe that their circumcision is best than our tradition". Another elder indicated that "MMC is not good because people who do this procedure look down at the people who do this at the mountains, but the truth is at the hospital females are involved and this is a disgrace to our tradition boys learn nothing as compared to those who did the procedure at the mountains". Traditional leaders and some community elders have rejected the circumcision act precisely because, like all other legislation, it diverts the power to administer the custom from traditional leaders and communities to the medical personnel (Ngeketo, 2008).

\section{Sub-theme: Less pain experienced}

The findings of this present study revealed that participants experienced less pain during medical male circumcision this was evidenced by elder 4 who said...."I even sent my two boys to the clinic to be circumcised, believe me it was a painless easy experience" Elder 3 in support of less pain experienced when undergoing MMC said "I don't know much about this MMC but I understand they give them medication to prevent them from feeling the pain, that is why they are not regarded as real men. Real men do not fear pain." Varying degrees of circumcision-related pain ranging from minimal to acceptable were observed up to six weeks post the procedure (Freeman, Spencer, Drongowski, Vandeven, Apgar, \& Teitelbaum, 2014). 


\section{Discussion}

The results pointed out two different opinions from the participants. The opinions include the fact that medical male circumcision is regarded as a commendable method. Males who opt for medical circumcision are to experience less pain during the procedure because they are given anesthetic during the procedure. The study conducted by Wambara, Mwanga, Mosha, Mshana, Mosha, and Changalucha (2011) indicated the shift of preference from traditional male circumcision to medical male circumcision due to the fact that there is no pain. It also indicated the need for the national male circumcision programme to meet the minimum quality of care for male medical circumcision in the clinical setting to minimize adverse events that might drive the clients away.

The study results also indicated that the safety of males undergoing medical circumcision is guaranteed due to the fact that the procedure is performed by trained medical practitioners and in a clean environment. Contrary to the study findings, the study conducted by Tarimo et al. (2012) indicated that there is worry that unsterile instruments may be used in hospitals as well in the hospital setting suggests that informants may avoid MMC even when it is promoted as the safest service. This indicates that there is a need to monitor the safety of the procedure in the hospital. However, a study conducted in Kenya indicates that MMC can be provided safely to adult men (Bailey et al., 2007).

The participants pointed out the fact that medical male circumcision undermines the black culture. This was supported by the study conducted by Wambara et al., (2011) which indicated that males circumcised in the traditional setting are initiated into manhood and prepared to take social responsibilities. It was further indicated that male circumcision performed in the traditional setting preserve the socio-cultural importance of the circumcision process. There is a direct stigma towards those undergoing MMC, arguing that circumcisions conducted in a clinic do not confer the status of manhood, unlike the tougher traditional version long practiced by Xhosa pre-initiates (Mathew, 2012).

\section{Conclusion}

The findings revealed mixed views regarding medical male circumcision. Some of the community elders commend MMC because it a safe and less painful procedure to undergo while others do not recommend it because it undermines the culture of black people.

\section{Recommendations}

The study, therefore, recommends that awareness campaigns should be conducted to inform the community about the importance of medical male circumcision. Department of Health should conduct workshops and training for traditional circumcisers and train them on how to maintain sterility during circumcision to avoid the initiates not having an infection.

\section{Competing Interests Statement}

The authors declare that there are no competing or potential conflicts of interest.

\section{References}

Bailey, R. C., Moses, S., Parker, C. B., Agot, K., Maclean, I., Krieger, J. N., ... \& Ndinya-Achola, J. O. (2007). Male circumcision for HIV prevention in young men in Kisumu, Kenya: a randomized controlled trial. Lancet, 369(9562), 643-656. https://doi.org/10.1016/S0140-6736(07)60312-2

Babbie, E., \& Mounton, J. (2012). The practice of social research. Cape Town: Oxford University of Press.

Brink, H. L., Van der Walt, C., \& Rensburg, G. (2012). Fundamental of research methodology for health professionals (3rd ed.). Cape Town, South Africa.

Creswell, J. W. (2009). Research design: qualitative and quantitative approach. USA: SAGE Publishers.

De Vos, A. S., Strydorm, H., Fouché, C. B., \& Delport, C. S. L. (2011). Research at Grassroots for the social science and human service professions (3rd ed,). Pretoria: Van Schaik Publishers.

Freeman, J. J., Spencer, A. U., Drongowski, R. A., Vandeven, C. J., Apgar, B., \& Teitelbaum, D. H. (2014). Newborn circumcision outcomes: Are parents satisfied with the results? Pediatric Surgery International, 30, 333-338. https://doi.org/10.1007/s00383-013-3430-5

Gwandure, C. (2011). The ethical concerns of using medical male circumcision in HIV prevention in sub-Saharan Africa. South African Journal of Bioethics and Law, 4(2), 89. https://doi.org/10.7196/SAJBL.169

Kripke, K., Okello, V., Maziya, V., Benzerga, W., Mirira, M., Gold, E., et al. (2016). Voluntary Medical Male Circumcision for HIV Prevention in Swaziland: Modeling the Impact of Age Targeting. PLoS ONE, 11(7). 
ttps://doi.org/10.1371/journal.pone.0156776

Mathew, W. (2012). Cutting into perceptions: Investigating men's understanding of protection - through medical male circumcision for HIV prevention, in Durban, KwaZulu-Natal (MSocSci dissertation. Durban: University of KwaZulu-Natal, November 2012).

Mavundla, T. R., Netswera, F. G., Toth, F., Bottman, B., \& Tenge, S. (2010). How boys became dogs: stigmatization and marginalization of Uninitiated Xhosa males in East London, South Africa. Qualitative Health Research, 20(7), 931-941. https://doi.org/10.1177/1049732310363804

Morris, B. J., \& Cox, G. (2012) Current medical evidence supports male circumcision, a surgical guide to circumcision. Bioessays, 83(1), 201-231. https://doi.org/10.1007/978-1-4471-2858-8_19

Morris, B. J., Bailey, R. C., Klausner, J. D., et al. (2012). Review: A critical evaluation of arguments opposing male circumcision for HIV prevention in developed countries. AIDS Care, 24(12), 1565-1575. https://doi.org/10.1080/09540121.2012.661836

Ngeketo, A. (2008). Xhosa male circumcision at the crossroads: Response by the government, traditional authority, and community to circumcision-related injuries and death in Eastern Cape Province (The Masters Dissertation University of the Western Cape).

Peltzer, K. (2009). Utilization and practice of traditional/complementary/alternative medicine in South Africa. Journal of Social and Development in South Africa, 6(2), 175-185.

Savestani, A. S., Bufumbo, L., Geiger, J. D., \& Sienko, K. H. (2012). Traditional male circumcision in Uganda: A qualitative focus group discussion Analysis. PLOS One Journals. https://doi.org/10.1371/journal.pone.0045316

Sgaier, S. K., Reed, J. B., Thomas, A., \& Njeuhmeli, E. (2014). Achieving the HIV Prevention Impact of Voluntary Medical Male Circumcision: Lessons and Challenges for Managing Programs. PLoS Med, 11(5): e1001641. https://doi.org/10.1371/journal.pmed.1001641

Tarimo, E. A. M., Francis, J. M., Kakoko, D., et al. (2012). The perceptions of male circumcision as a preventive measure against HIV infection and considerations in scaling up of the services: a qualitative study among police officers in Dar es Salaam, Tanzania. BMC Public Health. http://www.biomedcentral.com/1471-2458/12/529

Wambura, M., Mwanga, J. R., Mosha, J. F., Mshana, G., Mosha, F., \& Changalucha, J. (2011). Acceptability of medical male circumcision in the traditionally circumcising communities in Northern Tanzania. BMC public health, 11, 373. https://doi.org/10.1186/1471-2458-11-373

WHO. (2009). Male circumcision situational analysis. Geneva: WHO publishers.

WHO and UNAIDS. (2007). Voluntary medical male circumcision for HIV prevention. Geneva: WHO publishers WHO and UNAIDS. (2008). Manual for male circumcision under local anesthesia. Geneva: WHO Publishers.

WHO and UNAIDS. (2010). Neonatal and child male circumcision: A global review. Geneva: WHO publishers.

\section{Copyrights}

Copyright for this article is retained by the author(s), with first publication rights granted to the journal.

This is an open-access article distributed under the terms and conditions of the Creative Commons Attribution license (http://creativecommons.org/licenses/by/4.0/). 